\title{
Comparaison de la disponibilité de formes orales commercialisées utilisables en pédiatrie en France et au Canada
}

\author{
Jennifer Corny, Denis Lebel, Benoit Bailey, Sonia Prot-Labarthe et Jean-François Bussières
}

\section{INTRODUCTION}

T e manque de formes orales commercialisées utilisables en Lédiatrie est un problème récurrent et d'actualité. En Europe, la Paediatric Regulation (2006) de l'Union européenne a instauré les plans d'investigation pédiatrique (PIP), dont une section porte sur le développement de ces formes orales adaptées aux enfants ${ }^{1}$. Aux États-Unis, le Best Pharmaceuticals for Children Act (2002, révisé en 2007)² a souligné la nécessité de réaliser des études cliniques en pédiatrie, qui étayent l'usage de formes adaptées à ce type de patients. Récemment, en 2013, la Food and Drug Administration (FDA) a mis en place les pediatric study plans ${ }^{3}$, similaires aux PIP européens. Au Canada, des initiatives gouvernementales ont été prises afin d'inciter les laboratoires pharmaceutiques à développer ces formes orales, sans leur imposer d'obligations règlementaires, contrairement aux autres pays ${ }^{4}$. En 2006, une étude menée en France et au Canada ${ }^{5}$ avait soulevé les différences entre les formes orales commercialisées utilisables en pédiatrie et disponibles en France et au Canada. Elle a révélé qu'au total, 191 présentations (127 dénominations communes [DC]) étaient disponibles en France, contre 151 présentations (94 DC) au Canada.

La forme orale pédiatrique idéale est définie par le Pediatric Advisory Committee (FDA) comme " un médicament sans goût ou au goût masqué, avec un nombre d'excipients minimal, permettant une incrémentation facile des doses, se dissolvant oralement ou étant facile à avaler ou à dissoudre dans un faible volume de liquide et stable à la chaleur, à l'humidité et à la lumière " [traduit de l'anglais] ${ }^{6}$. Bien que le développement de formes orales répondant à tous ces critères soit complexe, il est indispensable d'avoir à disposition des médicaments favorisant une meilleure prise en charge des enfants et limitant les utilisations sans avis de conformité ou sans autorisation de mise sur le marché, source de risque accru d'erreurs de dose ou d'exposition à des excipients ou à des principes actifs n'ayant pas été étudiés pour cette population.

Dans ce contexte, nous nous sommes intéressés spécifiquement à la disponibilité des formes orales commercialisées utilisables en pédiatrie et disponibles en France et au Canada en 2015 (DC, laboratoires pharmaceutiques concernés et types de formes orales disponibles).

\section{DESCRIPTION}

Étude descriptive transversale. L'objectif principal était de décrire le profil des formes orales commercialisées utilisables en pédiatrie en France et au Canada. Les objectifs secondaires étaient de décrire les laboratoires pharmaceutiques concernés, le type de formes orales et de détailler quelques exemples des différences observées.

\section{Extraction des données}

Pour réaliser cette étude, nous avons extrait la liste des formes orales commercialisées utilisables en pédiatrie et disponibles dans ces deux pays. Les extractions, réalisées le 13 janvier 2015, permettaient de relever les formes orales potentiellement utilisables en pédiatrie et non les formes orales homologuées en pédiatrie.

Les données concernant la France ont été extraites de la base de données Thériaque ${ }^{7}$, à l'aide des filtres suivants appliqués à l'ensemble des médicaments commercialisés : « voie orale " ET « granules » ou " granulés » ou « liquide » ou «poudre " ou «sirop » ou "solution » ou "suspension ». Les médicaments homéopathiques, de phytothérapie et sous autorisation temporaire d'utilisation ont été exclus.

Les données portant sur le Canada ont été extraites de la base de données des produits pharmaceutiques de Santé$\mathrm{Canada}^{8}$, à l'aide des filtres suivants appliqués à l'ensemble des médicaments homologués : «voie orale » $\mathrm{ET}$ « comprimé 
pour suspension ", "gouttes ", "granules ", " granules pour solution ", " granules pour suspension ", " granules pour suspension, à libération prolongée ", "granules pour suspension, à libération retardée ", " poudre ", " poudre (effervescente) ", " poudre (à dose mesurée) ", " poudre (à libération prolongée) ", " poudre pour solution ", " poudre pour suspension ", " poudre pour suspension, à libération prolongée ", " sirop ", " sirop (à libération prolongée) ", " solution ", " solution (à libération prolongée) ", " suspension ", " suspension (à libération prolongée) ». Cette base de données n’inclut pas les produits de santé naturels ni les remèdes homéopathiques.

Ainsi, seuls les produits ayant un statut de médicament et commercialisés ont été pris en compte : les médicaments obtenus par des programmes d'accès spéciaux ont été exclus.

Pour les deux pays, les chercheurs ont pris en compte le nombre total de médicaments à usage humain commercialisés à l'aide des mêmes bases de données (toutes formes confondues). La collecte a porté sur les variables suivantes : nom commercial, dénomination commune, laboratoire pharmaceutique, forme, dosage, type de médicament (princeps ou médicament de marque/ générique), classe thérapeutique selon la classification Anatomical Therapeutic Chemical (ATC) $4^{\mathrm{e}}$ niveau (p. ex. J05AH) et type de délivrance pharmaceutique (prescription/vente libre).

Chaque concentration ou dosage était considéré comme une forme orale différente.

\section{Analyse des données}

Les chercheurs ont réalisé des tests du $\chi^{2}$ et des statistiques descriptives sur les données collectées.

\section{ÉVALUATION}

Ils ont trouvé en France 825 présentations de formes orales commercialisées utilisables en pédiatrie, correspondant à 272 DC et à 152 classes thérapeutiques ATC.

Ils ont trouvé au Canada 543 présentations, correspondant à 131 DC et à 93 classes thérapeutiques ATC. Cette différence était statistiquement significative $(p<0,001)$.

\section{Tableau 1. Profil des formes orales commercialisées utilisables en pédiatrie et disponibles en janvier 2015 et les cinq premières classes thérapeutiques et dénominations communes comportant le plus de formes orales utilisables en pédiatrie, disponibles en France et au Canada}

\begin{tabular}{|c|c|c|c|}
\hline Variables & France & Canada & Test $\chi^{2}$ \\
\hline $\begin{array}{l}\text { Nombre total de formes disponibles } \\
\text { (toutes voies confondues) }\end{array}$ & 12689 & 14413 & $\mathrm{NA}$ \\
\hline $\begin{array}{l}\text { Nombre de présentations orales } \\
\text { pédiatriques disponibles } \\
\text { (\% du nombre total) }\end{array}$ & $825(6,5 \%)$ & $543(3,8 \%)$ & $p<0,001$ \\
\hline Nombre de dénominations communes & 272 & 131 & NA \\
\hline Nombre (\%) de présentations & & & $p=0,03$ \\
\hline Sur prescription & $432(52,4 \%)$ & $252(46,4 \%)$ & \\
\hline En vente libre & $393(47,6 \%)$ & $291(53,6 \%)$ & \\
\hline Nombre (\%) de présentations & & & $p=0,17$ \\
\hline Princeps & $530(64,2 \%)$ & $368(67,8 \%)$ & \\
\hline Génériques & $295(35,8 \%)$ & $175(32,2 \%)$ & \\
\hline Laboratoires & 123 & 79 & NA \\
\hline $\begin{array}{l}\text { Nombre de classes thérapeutiques } \\
\text { (ATC niveau 4) }\end{array}$ & 152 & 93 & NA \\
\hline $\begin{array}{l}5 \text { classes thérapeutiques } \\
\text { (ATC niveau 4) comportant le plus } \\
\text { de formes orales pédiatriques } \\
\text { (nombre de présentations) }\end{array}$ & $\begin{array}{l}\text { R05CB : Médicaments contre le rhume } \\
\text { et la toux - mucolytiques }(n=71) \\
\text { R05DA : Médicaments contre le rhume } \\
\text { et la toux - alcaloïdes de l'opium et } \\
\text { dérivés }(n=48) \\
\text { J01CA : Antibactériens à usage } \\
\text { systémique - pénicillines à large } \\
\text { spectre }(n=40) \\
\text { A06AD : Médicaments contre la } \\
\text { constipation - laxatifs osmotiques } \\
\text { ( } n=39) \\
\text { J01CR : Antibactériens à usage } \\
\text { systémique - associations de } \\
\text { pénicillines, inhibiteurs de } \\
\text { B-lactamases inclus }(n=36)\end{array}$ & $\begin{array}{l}\text { N02BE : Analgésiques - anilides ( } n=82 \text { ) } \\
\text { R05DA : Médicaments contre le rhume } \\
\text { et la toux - alcaloïdes de l'opium } \\
\text { et dérivés }(n=61) \\
\text { R05FA : Médicaments contre le rhume } \\
\text { et la toux - dérivés de l'opium et } \\
\text { expectorants }(n=46) \\
\text { J01FA : Antibactériens à usage } \\
\text { systémique - macrolides ( } n=27) \\
\text { J01CA : Antibactériens à usage } \\
\text { systémique - pénicillines à large } \\
\text { spectre }(n=21)\end{array}$ & NA \\
\hline $\begin{array}{l}5 \text { dénominations communes } \\
\text { internationales comportant le plus } \\
\text { de formes orales pédiatriques } \\
\text { (nombre de présentations) }\end{array}$ & $\begin{array}{l}\text { Amoxicilline }(n=40) \\
\text { Amoxicilline/acide clavulanique }(n=36) \\
\text { Paracétamol (acétaminophène) }(n=27) \\
\text { Oxomémazine }(n=20) \\
\text { Cefpodoxime }(n=20)\end{array}$ & $\begin{array}{l}\text { Paracétamol (acétaminophène) }(n=82) \\
\text { Amoxicilline }(n=20) \\
\text { Docusate de sodium }(n=18) \\
\text { Azithromycine }(n=15) \\
\text { PEG } 3350(n=13)\end{array}$ & NA \\
\hline
\end{tabular}

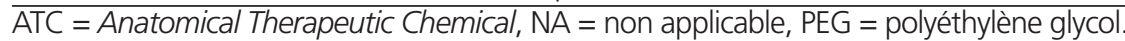


Le tableau 1 décrit les formes orales commercialisées utilisables en pédiatrie, disponibles en France et au Canada, ainsi que les cinq classes thérapeutiques et les cinq DC avec le plus de formes orales commercialisées.

Nous nous sommes intéressés plus particulièrement aux médicaments contre les infections à VIH et aux antiépileptiques, car ils représentent un enjeu majeur pour les enfants en raison de l'importance de poursuivre le traitement sans interruption et de leur marge thérapeutique étroite. En ce qui concerne les médicaments contre les infections à VIH (classes ATC J05AE, J05AF, J05AG, J05AR), nous avons trouvé 17 formes orales commercialisées utilisables en pédiatrie en France (15 DC) contre 9 (8 DC) au Canada. En ce qui concerne les médicaments antiépileptiques (classes ATC N03), on en a trouvé 29 en France (14 DC) contre 16 au Canada (7 DC). Les DC concernées sont répertoriées au tableau 2.

De plus, le nombre moyen de formes orales par laboratoire pharmaceutique était de 6,7 présentations par laboratoire en France et de 6,87 au Canada. Le tableau 3 présente une synthèse de ces données.

Le tableau 4 montre le profil des différents types de formes orales disponibles. En France, la forme orale la plus représentée était la solution orale ( $n=292,35,4 \%$ des formes) versus le sirop au Canada ( $n=200,36,8 \%$ formes).

\section{CONSÉQUENCES SUR LA PRATIQUE}

Cette étude a révélé de nombreuses différences de disponibilité de formes orales commercialisées utilisables en pédiatrie entre la France et le Canada. Ces différences concernaient le nombre de formes disponibles, de dénominations communes, de classes thérapeutiques ATC (niveau 4) et de laboratoires pharmaceutiques concernés.

\section{Nombre de formes orales pédiatriques disponibles en France et au Canada}

En France, 825 présentations orales utilisables en pédiatrie ont été trouvées contre 543 au Canada, ce qui représente respectivement $6,5 \%$ versus 3,8 \% de l'ensemble des médicaments commercialisés en France et au Canada. Parallèlement, en date du $1^{\text {er }}$ janvier 2016, en France, la proportion d'enfants entre zéro et 18 ans révolus était de $23,4 \%{ }^{9}$, alors qu'au $1^{\text {er }}$ juillet 2015 , elle était de $21,9 \%$ au Canada ${ }^{10}$. Ainsi, le facteur démographique ne peut être invoqué pour expliquer cette différence.

\begin{tabular}{|c|c|}
\hline Catégorie & Canada \\
\hline \multirow[t]{2}{*}{ Anti-VIH } & $\begin{array}{l}\text { Abacavir, darunavir, fosamprénavir, lamivudine, lopinavir/ritonavir, } \\
\text { ritonavir, zidovudine }\end{array}$ \\
\hline & $\begin{array}{l}\text { Didanosine, efavirenz, emtricitabine, } \\
\text { entecavir, nevirapine, stavudine, } \\
\text { tenofovir, tipranavir }\end{array}$ \\
\hline \multirow[t]{2}{*}{ Antiépileptiques } & $\begin{array}{l}\text { Acide valproïque, carbamazepine, ethosuximide, oxcarbazepine, } \\
\text { phenytoine, stiripentol, vigabatrin }\end{array}$ \\
\hline & $\begin{array}{l}\text { Clonazepam, felbamate, lacosamide, } \\
\text { levetiracetam, phenobarbital, } \\
\text { pregabaline, rufinamide }\end{array}$ \\
\hline
\end{tabular}

Tableau 3. Profil et noms des laboratoires pharmaceutiques fabriquant des formes orales pédiatriques en France et au Canada*

\begin{tabular}{|c|c|c|}
\hline Variables & France & Canada \\
\hline Nombre de laboratoires & 123 & 79 \\
\hline $\begin{array}{l}\text { Nombre moyen de présentations } \\
\text { par laboratoire }\end{array}$ & 6,7 & 6,87 \\
\hline \multirow[t]{9}{*}{$\begin{array}{l}10 \text { laboratoires fabriquant le plus de } \\
\text { formes orales utilisables en pédiatrie } \\
\text { (nombre de présentations) }\end{array}$} & $\begin{array}{l}\text { Sanofi-Aventis }(n=86) \\
\text { Mylan }(n=44) \\
\text { Sandoz }(n=32)\end{array}$ & $\begin{array}{l}\text { Teva }(n=53) \\
\text { Pharmascience }(n=52) \\
\text { McNeil }(n=42)\end{array}$ \\
\hline & Teva $(n=31)$ & Novartis $(n=34)$ \\
\hline & $\operatorname{Biogaran}(n=31)$ & Pfizer $(n=27)$ \\
\hline & $\operatorname{Arrow}(n=26)$ & Jamp Pharma $(n=25)$ \\
\hline & EG Labo $(n=24)$ & Apotex $(n=24)$ \\
\hline & Bristol-Myers Squibb $(n=22)$ & Pendopharm $(n=24)$ \\
\hline & Pierre-Fabre $(n=20)$ & Trillium Health Care $(n=19)$ \\
\hline & Zambon $(n=18)$ & Pharmetics $(n=12)$ \\
\hline & Autres ( $n=491$ pour 113 laboratoires) & Autres ( $n=231$ pour 69 laboratoires) \\
\hline
\end{tabular}


This single copy is for your personal, non-commercial use only.

For permission to reprint multiple copies or to order presentation-ready copies for distribution, contact CJHP at cjhpedit@cshp.ca

Tableau 4. Profil des types de formes orales utilisables en pédiatrie, commercialisées en France et au Canada

\begin{tabular}{|c|c|c|}
\hline \multirow[b]{2}{*}{$\begin{array}{l}\text { Types de formes orales commercialisées } \\
\text { et utilisables en pédiatrie }\end{array}$} & \multicolumn{2}{|c|}{ Pays; nombre (\%) de présentations } \\
\hline & France & Canada \\
\hline Solution orale & $292 \quad(35,4)$ & $105 \quad(19,3)$ \\
\hline Suspension orale & $188 \quad(22,8)$ & $169 \quad(31,1)$ \\
\hline Poudre (sachet/pot) & $151 \quad(18,3)$ & $63 \quad(11,6)$ \\
\hline Granules (sachet/pot) & $(9,6)$ & $(1,1)$ \\
\hline Sirop & $115 \quad(13,9)$ & $200 \quad(36,8)$ \\
\hline Total & $825(100,0)$ & $543(100,0)$ \\
\hline
\end{tabular}

Dans l'étude publiée en 2008 par Prot-Labarthe et collab. $^{5}, 191$ formes orales pédiatriques avaient été trouvées en France et 154 au Canada. En presque dix ans, le nombre de présentations a donc considérablement augmenté en France et au Canada. Il est toutefois important de souligner que la méthode utilisée dans l'étude de Prot-Labarthe et collab. ${ }^{5}$ ne permettait pas de recenser tous les produits du marché, la collecte de données étant limitée aux données correspondant aux ententes d'achats groupés en France et au Canada, ce qui reflétait les besoins du marché hospitalier mais pas nécessairement des besoins en pharmacie communautaire.

De plus, la Paediatric Regulation de l'Union européenne ${ }^{1}$ adoptée en 2006, a instauré les PIP obligeant les laboratoires à réaliser des études cliniques pour les enfants si le médicament peut être administré à cette population. Dans ces PIP, le laboratoire doit notamment décrire la forme galénique qui sera utilisée " afin de rendre son utilisation plus acceptable, facile, sécuritaire et efficace pour l'ensemble des sous-populations pédiatriques " [traduit de l'anglais] ${ }^{1}$. Depuis leur mise en place, 1371 décisions ont été rendues par l'European Medicines Agency (en date du 25 octobre 2016) ${ }^{11}$. Au Canada, aucune mesure gouvernementale n'est en ouvre pour obliger les laboratoires à faire de même. Ceci a pu participer à la différence notée près de dix ans après l'étude de Prot-Labarthe et collab. ${ }^{5}$

\section{Nombre de dénominations communes pour lesquelles une forme orale pédiatrique est disponible en France et au Canada}

En ce qui concerne le nombre de DC pour lesquelles une forme orale commercialisée utilisable en pédiatrie est disponible, 272 ont été trouvées en France contre 131 au Canada. La Paediatric Regulation de l'Union européenne a probablement joué un rôle conséquent dans cette différence de disponibilité.

Toutefois, nous pensons que la plus grande variété de DC de certaines classes thérapeutiques est davantage liée aux stratégies commerciales (centralisation des autorisations de mise sur le marché en Europe, ce qui crée un marché de taille supérieure ${ }^{12}$ ), aux habitudes de prescription mettant en valeur un plus grand nombre de médicaments ou aux recommandations d'utilisation de médicaments en pédiatrie.

Parmi les cinq DC comportant le plus grand nombre de formes orales utilisables en pédiatrie, on trouve des médicaments communs : paracétamol (acétaminophène) et amoxicilline. Ces cinq DC représentent respectivement $1,1 \%$ et $1 \%$ de toutes les formes commercialisées en France et au Canada.

Notre analyse met en évidence un déficit préoccupant de DC commercialisées utilisables en pédiatrie au Canada. Par exemple, certains antiépileptiques ou antiviraux sont disponibles sous une présentation orale pédiatrique en France (tableau 2). Leur indisponibilité au Canada soulève des interrogations sur l'équité d'accès aux médicaments et oblige les pharmaciens des établissements de santé à réaliser davantage de préparations magistrales, dont la stabilité et la pharmacocinétique sont parfois méconnues, ce qui rend leur utilisation pour les enfants d'autant plus délicate .

L'étude réalisée en 2006 par Prot-Labarthe et collab. ${ }^{5}$, avait rapporté 127 DC en France contre 94 au Canada. La méthodologie étant différente, il est impossible de procéder à une comparaison directe de ces deux études. Cependant, il semble qu'en France, les mesures incitatives des PIP puissent avoir joué un rôle sur l'augmentation supérieure à celle du Canada du nombre de DC bénéficiant d'une forme orale commercialisée utilisable en pédiatrie.

\section{Classes thérapeutiques ATC pour lesquelles des formes orales pédiatriques sont commercialisées en France et au Canada}

En ce qui concerne les classes thérapeutiques prises en compte, on note des différences majeures. En France, 152 classes thérapeutiques selon la classification ATC étaient représentées contre 93 au Canada.

On remarque des différences notables dans l'usage des médicaments pour le traitement des infections à VIH chez les enfants. En France, on trouve 17 formes (15 DC) de ces médicaments contre 9 (8 DC) au Canada (tableau 2). La Société canadienne de pédiatrie suggère le suivi des recommandations du National Institutes of Health portant sur la prise en 
charge du VIH chez les enfants (dernière mise à jour date de février 2014). Elles incluent notamment en première ligne des médicaments non disponibles sous une forme adaptée aux enfants ${ }^{13}$. Un deuxième exemple est le cas des antiépileptiques. En France, 29 formes sont disponibles (14 DC), contre 16 au Canada (7 DC). Les DC sont détaillées dans le tableau 2. Parmi celles non commercialisées au Canada sous une forme utilisable en pédiatrie, certaines constituent un arsenal thérapeutique essentiel au traitement des différents types d'épilepsie en pédiatrie. Un des médicaments proposés comme première intention lors d'un état de mal épileptique de l'enfant est le midazolam (voie orale) ${ }^{14}$. Au Canada, aucune forme orale de midazolam n'est disponible, contrairement à la France, où des seringues préalablement remplies de quatre dosages différents sont disponibles depuis $2011^{15}$.

\section{Type de formes orales pédiatriques disponibles en France et au Canada}

Enfin, le type de formes orales disponibles dans ces deux pays et leurs proportions respectives peuvent varier. Par exemple, on trouve une proportion plus importante de sirop au Canada qu'en France (respectivement 36,8 \% et 13,9\% des présentations orales pédiatriques disponibles), et l'inverse se vérifie pour les solutions buvables (19,3\% au Canada et 35,4\% en France). Les études portant sur les formes orales pédiatriques sont de plus en plus nombreuses, et le choix d'une forme ou d'une autre est notamment dicté par les propriétés physicochimiques et le goût de la substance active.

Différentes initiatives, notamment sur le plan européen sous l'impulsion du consortium European Paediatric Formulation Initiative (EuPFI), travaillent à l'amélioration des connaissances sur la mise au point de formules plus adaptées aux enfants ${ }^{16}$. Des initiatives locales, comme la création du premier centre de formulations pédiatriques au Canada, sont également notables et permettront d'enrichir les données disponibles ${ }^{17}$.

Dans le rapport Améliorer les médicaments pour enfants au Canada du Conseil des académies canadiennes ${ }^{18}$, les experts mettent en évidence qu' "aux États-Unis et dans l'Union européenne, la recherche sur des médicaments pédiatriques est encouragée, obligatoire et surveillée selon des modalités qui constituent des leçons utiles pour le Canada ". Notre étude confirme l'inégalité importante existant entre le marché français et canadien. Il semble inacceptable que certaines populations pédiatriques n'aient pas un accès comparable aux formes orales appropriées. Avec la mondialisation des marchés, les cliniciens devraient s'étonner de ces écarts et les dénoncer, les productions pharmaceutiques étant souvent délocalisées et regroupées au sein d'un nombre limité d'usines à travers le monde. Lorsqu'une forme galénique orale pédiatrique est réalisable, elle devrait être accessible à tous les pays.

\section{LIMITES}

L'étude menée ne portait que sur la France et le Canada. Une analyse de disponibilité à l'échelle de l'ensemble des pays européens et des États-Unis pourrait donner un portrait plus global de la question. Les filtres utilisés dans les bases de données permettaient de recenser les différentes formes potentiellement utilisables en pédiatrie, et non celles réellement utilisées en pédiatrie. Notons que les comprimés à désintégration orale rapide (c.-à-d. lyophilisat) et les comprimés effervescents ont été exclus de notre analyse. Ces deux formes pharmaceutiques peuvent aussi être utilisées en pédiatrie mais ne permettent pas d'adapter les doses. Par ailleurs, des différences de statut entre les deux pays peuvent constituer un biais (commercialisation versus programme d'accès spécial, statut de médicament ou produit de santé naturel, etc.). Lévaluation de la pertinence des formes galéniques disponibles pourrait permettre de déterminer avec plus de précision les patients lésés par l'indisponibilité de présentations orales pédiatriques.

\section{CONCLUSION}

Cette étude descriptive a permis de mettre en évidence que la France bénéficie de la commercialisation d'un nombre bien plus important que le Canada de formes orales utilisables en pédiatrie (nombre de dénominations communes, classes thérapeutiques, laboratoires pharmaceutiques concernés). On note donc que des écarts importants existent entre les deux pays étudiés concernant l'accessibilité aux médicaments destinés aux enfants, et ce, malgré l'augmentation de la disponibilité de formes pédiatriques depuis dix ans. La règlementation en vigueur et les incitations gouvernementales peuvent avoir des retombées directes sur ces résultats. Par ailleurs, afin de renforcer la sécurité d'utilisation des médicaments en pédiatrie, des initiatives, telles que celles de l'EuPFI, feront certainement progresser cette question d'actualité.

\section{Références}

1. Paediatric Regulation (Regulation [EC] No 1901/2006 of the European Parliament and of the Council of 12 December 2006 on medicinal products for paediatric use and Amending Regulation [EEC] No 1768/92, Directive 2001/20/EC, Directive 2001/83/EC and Regulation [EC] No 726/2004). Parlement européen; 2006. Publié au : http://ec.europa.eu/health/files/ eudralex/vol-1/reg_2006_1901/reg_2006_1901_en.pdf. Consulté le 14 juillet 2016.

2. Food and Drug Administration Amendments Act of 2007. Title V: Best Pharmaceuticals for Children Act of 2007. H.R. 3580, 110th Congress, Public Law 100-85. Publié au : http:/www.fda.gov/downloads/Drugs/ DevelopmentApprovalProcess/DevelopmentResources/UCM049870.pdf. Consulté le 14 juillet 2016.

3. Pediatric study plans. Content of and process for submitting initial pediatric study plans and amended initial pediatric study plans. Guidance for industry [draft]. Silver Spring (MD) : U.S. Department of Health and Human Services, Food and Drug Administration, Center for Drug Evaluation and Research, Center for Biologics Evaluation and Research; 2016. Publié au : http:// 
www.fda.gov/downloads/drugs/guidancecomplianceregulatoryinformation/ guidances/ucm360507.pdf. Consulté le 14 juillet 2016.

4. À propos de Santé Canada : Bureau des initiatives pédiatriques; 2010. Publié au : http://www.hc-sc.gc.ca/ahc-asc/branch-dirgen/hpfb-dgpsa/opi-bip/ index-fra.php. Consulté le 25 octobre 2016.

5. Prot-Labarthe S, Bussières JF, Barret P, Bourdon O, Brion F. Lack of commercial oral drug formulations for children in two hospital group purchasing agreements in France and Canada. Int J Pharm Compound. 2008; 12(4):365-7.

6. Zajicek A. Pediatric formulations: NIH-FDA cooperative efforts. Food and Drug Administration, Pediatric Advisory Committee; 2012. Publié au : http://www.fda.gov/downloads/AdvisoryCommittees/Committees MeetingMaterials/PediatricAdvisoryCommittee/UCM289959.pdf. Consulté le 14 juillet 2016.

7. Thériaque [banque de données]. Paris (France) : Centre national hospitalier d'information sur le médicament; 2015. Publié au : http://www.theriaque.org/ apps/contenu/accueil.php. Consulté le 13 janvier 2015.

8. Base de données sur les produits pharmaceutiques. Ottawa $(\mathrm{ON})$ : Santé Canada; 2015. Publié au : http://webprod5.hc-sc.gc.ca/dpd-bdpp/indexfra.jsp. Consulté le 13 janvier 2015.

9. Population totale par sexe et âge au 1er janvier 2015, France. Dans : Bilan démographique 2016. Paris (France) : Institut national de la statistique et des études économiques; 2016. Publié au : http://www.insee.fr/fr/themes/ detail.asp?ref_id=bilan-demo. Consulté le 14 juillet 2016.

10. Population selon le sexe et le groupe d'âge. Ottawa $(\mathrm{ON})$ : Statistique Canada; 2016. Publié au : http://www.statcan.gc.ca/tables-tableaux/sum-som/102/ cst01/demo10a-fra.htm. Consulté le 14 juillet 2016.

11. Opinions and decisions on paediatric investigation plans. London (UK) : European Medicines Agency; 2016. Publié au : http://www.ema.europa.eu/ ema/index.jsp?curl=pages\%2Fmedicines\%2Flanding\%2Fpip_search.jsp\&mi $\mathrm{d}=W C 0 \mathrm{~b} 01 \mathrm{ac} 058001 \mathrm{~d} 129 \&$ searchkwByEnter=false\&alreadyLoaded=true\& isNewQuery=true\&startLetter=View+all\&keyword=Enter+keywords\&search Type=Invented+name\&taxonomyPath=\&treeNumber $=$. Consulté le 25 octobre 2016.

12. Breitkreutz J. European perspectives on pediatric formulations. Clin Ther. 2008;30(11):2146-54

13. La prise en charge des enfants exposés au VIH ou infectés par le VIH. Ottawa $(\mathrm{ON})$ : Société canadienne de pédiatrie; affichage le $1^{\text {er }}$ août 2010 ; reconduit

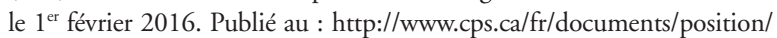
enfants-exposes-au-VIH-ou-infectes-par-le-VIH. Consulté le 1 1er mars 2017.

14. La prise en charge d'urgence du patient pédiatrique en état de mal épileptique convulsif généralisé. Ottawa $(\mathrm{ON})$ : Société canadienne de pédiatrie; affichage le $1^{\text {er }}$ février 2011; reconduit le $1^{\text {er }}$ février 2016. Publié au : http:// www.cps.ca/fr/documents/position/mal-epileptique-convulsif. Consulté le 14 juillet 2016.

15. Annexe I : Résumé des caractéristiques du produit. Monographie du Buccolam ${ }^{\circledR}$ (midazolam). Viropharma; 2011. Publié au : http:// www.ema.europa.eu/docs/fr_FR/document_library/EPAR_-_Product_ Information/human/002267/WC500112310.pdf. Consultéle 14 juillet 2016.
16. About us. London (UK): European Paediatric Formulation Initiative; 2016. Publié au : http://www.eupfi.org/about-eupfi/. Consulté le 25 octobre 2016.

17. Offrir aux enfants plus de médicaments sous une forme pharmaceutique adaptée à leurs besoins [communiqué de presse]. Montréal (QC) : Fondation CHU Sainte-Justine; le 22 février 2016. Publié au : http://www.fondationsainte-justine.org/fr/fondation/publications/nouvelles-communiques/ 2016/12374-le-chu-sainte-justine-creera-le-premier-centre-de-formulationspediatriques-au-canada. Consulté le 26 octobre 2016.

18. Le comité d'experts sur les produits thérapeutiques pour les nourrissons, les enfants et les adolescents. Améliorer les médicaments pour enfants au Canada. Ottawa (ON) : Conseil des académies canadiennes; 2014. Publié au : http://sciencepourlepublic.ca/uploads/fr/assessments\%20and\%20 publications\%20and $\% 20$ news\%20releases/therapeutics/therapeutics_ fullreportfr.pdf. Consulté le 14 juillet 2016.

Jennifer Corny, Pharm. D., M. Sc., travaille à I'Unité de recherche en pratique pharmaceutique, Centre hospitalier universitaire Sainte-Justine, Montréal (Québec)

Denis Lebel, B. Pharm, M. Sc., FCSHP, travaille au Département de pharmacie, Centre hospitalier universitaire Sainte-Justine, Montréal (Québec).

Benoit Bailey, M. D., travaille au Département des urgences, Centre hospitalier universitaire Sainte-Justine, Montréal (Québec).

Sonia Prot-Labarthe, Pharm. D., Ph. D., travaille au Département de pharmacie, Hôpital Robert-Debré, Assistance publique - Hôpitaux de Paris, Paris, France.

Jean-François Bussières, B. Pharm., M. Sc., FCSHP, travaille au Département de pharmacie et Unité de recherche en pratique pharmaceutique, Centre hospitalier universitaire Sainte-Justine, et à la Faculté de pharmacie, Université de Montréal, Montréal (Québec).

Intérêts concurrents : Aucun déclaré.

\section{Address correspondence to:}

Jennifer Corny

Unité de recherche en pratique pharmaceutique

Centre hospitalier universitaire Sainte-Justine

3175, chemin de la Côte Sainte-Catherine

Montréal QC H3T 1C5

Courriel : jennifercorny@yahoo.fr

Financement : Ce projet a notamment été réalisé avec le soutien du Réseau mère-enfant de la Francophonie (RMEF). 